\title{
The path to productive partnerships
}

\section{Research funders and institutions do too little to sustain the collaborations that they encourage.}

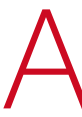
rich culture of collaboration is one of the valued treasures of the scientific community, and deservedly so. It is only natural that universities and funding agencies would want to encourage this approach. It is a shame that their support is only skin-deep.

Many funders have designed programmes that specifically encourage interdisciplinary or international collaborations. Such programmes can enhance funding for collaborations that do not fit easily into traditional grant programmes. More worrying are the initiatives that attempt social engineering, demanding that scientists include researchers from, say, a particular region in their funding application. As a result, efforts are wasted in collaborations that are motivated more by the funds themselves than by scientific necessity.

But most concerning of all is the fact that merely funding a culture of collaboration does too little to make it robust. More support by agencies, and foresight by researchers themselves, needs to be devoted to anticipating and coping with the traps that can lie ahead.

Financial incentives will not teach collaborators how to work as a team. Interdisciplinary endeavours by definition bring together researchers from diverse scientific cultures, with respective languages and priorities. Computer scientists, for instance, may think nothing of making their data freely available on the Internet. But in highly competitive biomedical fields, in which whispers heard at meetings can quickly be turned into papers bearing the eavesdroppers' names, sharing too soon can have unintended consequences.

Meanwhile, crossing political borders carries the risk of complications that can befuddle expert regulators, let alone inexperienced researchers. The rules regarding ownership of intellectual property vary from country to country, as do ethical regulations on experiments involving humans or animals. And when cases of misconduct arise, it is unclear which country or regulatory authority has jurisdiction (see page 686).

\section{Defining 'natural'}

\section{Visceral reactions to an act should not distract from the real ethical issues.}

ए rom an evolutionary perspective, we humans have good reason to be wary of things that seem to be 'unnatural'. Anything out of the ordinary can be dangerous. But the evolutionary origin of that response also guarantees that it will be guided more by emotion than by reason. Witness the reaction last week when Thomas Beatie, from Bend, Oregon, announced his pregnancy on the popular television talk show, Oprah.

Beatie, who was born female (and participated in beauty pageants), underwent hormone treatment and some gender-reassignment surgery ten years ago, but retained his reproductive organs. He stopped
These difficulties are not limited to large-scale, high-budget projects, yet researchers too often enter into the complex landscape of collaboration with little, if any, thought about the complications that can arise - even from a small-scale alliance (for examples, see page 682). The culture of informal collaboration feeds scientific innovation and productivity, but it must be balanced by good planning. Wise collaborators will map out their endeavours and responsibilities early in the relationship, keeping a written record of their agreement. These plans need not be limiting: they can and should be amended as the project progresses.

"Researchers too often enter into collaborations with little thought about the complications that can arise."

Meanwhile, an early conversation about data ownership and authorship can prevent the hostility that may arise when such issues are disputed later in the relationship. This discussion should include, from day one, any proposed industrial contacts and contracts. When publication of the results is the goal, reagents, methods and data used and developed during the collaboration need to be kept accessible to the researchers, even if licensed to a second party. Without a written agreement, access to these reagents cannot be ensured.

But expecting researchers themselves to invent such principles from scratch is a recipe for wasted effort. What is more, when researchers fall out, the burden of clearing up the mess can fall heavily on their institutions. Thus the institutions, and funding agencies too, should support collaborations not only with facilities and funds but also through logistical support in the form of guidelines, checklists, model agreements and training - or even counselling, if it should come to that. In that way they can help researchers and themselves avoid the pain, and benefit from the pleasures and insights that collaboration should bring.

taking hormones so that he and his wife, who cannot bear children, could pursue artificial insemination. Several doctors turned them down, but last week, the world watched as a baby-faced man with a thin beard and a growing paunch went for an ultrasound: the fetus was a girl. Oprah Winfrey was supportive as she nursed the nervous Beatie through a discussion of his personal realizations. So was the lesbian, gay, bisexual and transgender community. But other reactions were vitriolic, as when MSNBC's Joe Scarborough repeatedly commented that he was "going to be sick". Other such visceral responses were common on message boards and blogs on the Internet, where the situation was often held to be disgusting and unnatural.

And yet, when we consider this story with the reasoning parts of our brains, exactly what was so 'unnatural'? The longing to have a baby? That is a profoundly human desire, whether the prospective parents are male, female or transgendered. Or is it that Beatie has acted on his certainty that he is a man who happened to be born with- 
out a Y chromosome? Biologists have found that gender-straddling and gender-switching behaviours are not at all uncommon in the 'natural' world, either for humans or non-human animals (see page 678). True, modern biotechnology has considerably raised the stakes, and is allowing humans to manipulate their biological make-up to an ever-increasing degree. But it hasn't fundamentally changed the game. And its applications, however unsettling they may be to some people, are not, by definition, 'unnatural'.

This same question of 'natural' versus 'unnatural' also emerges this week in a very different context: an online poll that Nature started in January on the use of neuroenhancing drugs (see page 674). Respondents were asked to report on their non-medical use of drugs such as modafinil and methylphenidate to improve their concentration. These drugs can have mild effects, not all that different from caffeine (a natural substance) or other stimulants. But somehow the 'unnaturalness' of these drugs makes some people uneasy in a way that caffeine does not. The claim, repeated in many responses to our survey is that using such drugs, or any performance-enhancing drug, makes accomplishments somehow less worthy because they aren't natural. But again, what is 'natural'? Devices such as glasses, hearing aids, pacemakers and artificial hips are unnatural. Yet they are widely accepted as legitimate ways to enhance the human experience. By the same token, if drugs enhance performance on a standardized test, what is so 'natural' about prep courses designed to improve scores?

Ultimately, our visceral concept of what is 'natural' depends on what we are used to, and will continue to evolve as technology does. But in the meantime, we should not allow it to distract us from the rational consideration of deeper and more important ethical issues. In the case of Beatie and his wife, the elemental questions are the health, safety and emotional security of the child. Trying to decide such issues simply by fixating on a fluid and arbitrary definition is, by nature, silly.

\section{Ready or not}

\section{Transparency and honesty are essential if the genetic-testing industry is to live up to its potential.}

$\mathrm{N}$ avigenics, a California start-up company with solid backing, launched its flagship product this week. The Health Compass, a US $\$ 2,500$ genetic test, is being offered to consumers directly, over the Internet. It will scan DNA from a customer's saliva sample for a host of tiny variations and pronounce on the person's risk of developing 18 common medical conditions, including heart attack, prostate cancer and type 2 diabetes.

Why now? Because, as the company says in its corporate literature, "the science is ready. Genetic testing is ready to enter into common health-care practice."

Certainly genetic testing is here, ready or not. In the past year, genome-wide association studies have begun to pour out of labs, linking the blips in our genetic make-up to risks of developing particular medical conditions. Whether people would or could change their behaviour to ameliorate these risks remains unclear. But the ink on the research papers is barely dry before companies unveil commercial versions of the tests. Navigenics is simply the most recent; others include high-profile players such as 23 andMe in Mountain View California (see Nature 450, 11; 2007) and the Icelandic genomics company deCODE Genetics. They also encompass smaller operations such as Philadelphia-based Smart Genetics, which last month offered a genetic assessment of the risk of Alzheimer's disease. Rarely have basic discoveries morphed into a commercial product quite so swiftly.

Following almost as quickly are the concerns being raised about the use of such tests. Many of these worries have been around for some time, but now that the tests are here, these discussions have taken on a new urgency. If consumers are to reap the benefits that genetic testing can offer, they need understandable information about the basis, validity and limitations of the tests. One proposed structure for providing this information is a publicly accessible registry into which test-makers would be required to upload data about their tests and the studies that back them. This information should be updated as genetic risks are changed or refined, as inevitably they will be.

Such a registry should be international, harmonizing information in what will doubtless be an industry without borders. This approach seems preferable to stepped-up regulation by agencies such as the Food and Drug Administration (FDA), which - in addition to travelling at the snail's pace of bureaucracy rather than the lightning speed of burgeoning markets - could easily have the effect of driving lessthan-desirable players underground, where sub-standard tests will remain as easy to buy as black-market DVDs.

Many critics would say that a hands-off approach by the FDA is irresponsible. But on what basis should genetic tests be treated any differently from others done in government-certified clinical labs? Many of these tests - including almost all of those done by labs inhouse, rather than sent out to patients and doctors as kits - are not required to demonstrate clinical utility on a test-by-test basis. For that matter, why should genetic tests be treated differently from medical devices such as MRI scanners, which were left by the FDA to prove their utility and clinical validity to physicians and providers in the marketplace, rather than in pre-market assessments?

It would be naive to suggest that transparency will solve all problems, or to assume that the marketplace will separate the wheat from the chaff with unfailing accuracy and efficiency. But to advocate relatively light regulation does not mean turning a blind eye to the risks of such a strategy. It means taking seriously the presumption that people should be free to inform themselves and make their own choices, and that by doing so they may benefit not just themselves but also the overall pace of innovation. Should it become clear that the system is allowing harm, then enhanced regulation will be appropriate.

It is also worth noting that the scientists who have driven this revolution need to assume a prominent role in ensuring that its benefits are not mishandled. Those who start companies, or advise them, can and must lead the way in ensuring that their enterprises are transparent and valid. In the meantime, online shoppers who buy genetic tests would do well to keep asking themselves whether the science is, indeed, ready. 